\title{
Atmospheric Pressure Plasma Jet for Mass Spectrometry
}

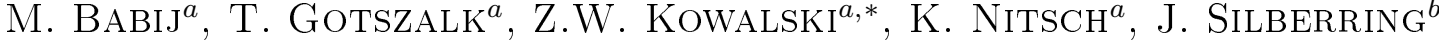 \\ AND M. SMOLUCH ${ }^{b}$ \\ ${ }^{a}$ Wrocław University of Technology, Wybrzeże Wyspiańskiego 27, 50-370 Wrocław, Poland \\ ${ }^{b}$ AGH University of Science and Technology, al. A. Mickiewicza 30, 30-059 Krakow, Poland
}

\begin{abstract}
The atmospheric pressure plasma is much advantageous over low pressure plasmas in various aspects, e.g. vacuum-free operation, relative low cost, flexibility of a continuous process. Among various plasmas generated in atmospheric pressure discharges there are cold plasma jets that represent a technology of great application promise (industry, medicine, biology). To generate low-temperature plasmas at atmospheric pressure the dielectric barrier discharge can be used. It is suitable for the atomization of volatile species and can also be served as a ionization source for ambient mass and ion mobility spectrometry. As the discharge is generated in a restricted electrode structure, a plasma jet (plume) is usually formed outside the electrode region (that provides spatial separation of the plasma generation and surface processing regions). The paper presents a source based on a plasma jet established at the end of a capillary dielectric barrier discharge at atmospheric pressure and its application to mass spectrometry. The structure of the jet generator consists of piezoelectric transformer and two concentric and symmetric electrodes, between which the working gas flows at definite rate. Besides the source description early results of spectroscopic analysis are also given.
\end{abstract}

DOI: 10.12693 /APhysPolA.125.1260

PACS: 52.50.Dg, 82.80.Ms

\section{Introduction}

Low temperature atmospheric pressure plasmas (APPs) have attracted a great interest in the last decades due to their interesting characteristics and wide ranges of applicability. They are routinely used in material processing [1] and, more recently, in industrial $[2,3]$ and biomedical applications $[4,5]$ (e.g. decontamination, cancer cell treatment, tooth treatment, blood coagulation, wound healing, electrosurgery, tissue engineering, surface modification of biocompatible materials, sterilization of heat sensitive materials and instruments). One of the attractive features of the plasmas in question is the ability to achieve enhanced plasma chemistry without the need for elevated gas temperatures. This is because these plasmas exhibit electron energies much faster than the heavier ions and the neutral species and have the opportunity to heat up to thousands of degrees before their environment heats up. Because the ions and the neutrals remain relatively cold (cooling of ions and uncharged molecules is more effective than energy transfer from electrons), the gas remains at low temperature and the plasma does not cause any thermal damage to objects it comes in contact. APPs are generated by a diversity of electrical discharges such as corona discharge, dielectric barrier discharge (DBD), micro hollow cathode discharge and so on, all having their own properties and use [6-12]. Among many atmospheric pressure plasma sources, the plasma jet system is more efficient than others (high charged particle and reactive species density in the plasma, low

\footnotetext{
* corresponding author; e-mail:
} zbigniew.w.kowalski@pwr.wroc.pl thermal temperature, usefulness of small size plasma jet in treating localized regions). Its characteristic opened up the possibility to use these plasmas for the treatment of heat-sensitive materials including biological cells and tissues [13-16].

The phenomenon of atmospheric pressure plasma jet (APPJ) can date back to 1960s. In the early 1990s, the study in applications of atmospheric plasma treatment has been gradually increased due to its relative low cost (cheaper and more convenient alternative in comparison with low-pressure plasmas) and attractive properties such as: (a) the reduced or no thermal damage to the substrate, (b) vacuum-free operation. Nonthermal APPJ can be generated in many different gases, different types of discharge setups under various power supplies and using different electrical excitations, different operating modes (filamentary, Townsend, glow, etc.). These include DC, $\mathrm{kHz}$ frequency pulsed and sinusoidal jets, radio frequency and microwave excited jets. Depending on jet electrode configuration, electrical excitation, chemical composition and flow rates of the carrier gas, plasma characteristics may differ significantly. An important characteristic of a jet configuration is spatial separation of surface processing and the plasma generation regions. That enables to vary and control plasma dynamics and reaction chemistry.

In the last years, researchers have reported a kind of APPJ generated by flowing gas through a dielectric tube wrapped with tubular electrodes supplied with pulsed or sinusoidal high voltage (in $\mathrm{kV}$ and $\mathrm{kHz}$ ranges). The DBD plasma jet is a classical example of that kind of nonthermal APPJ. A noble gas passes through the dielectric tube at certain flow rates and when the discharge is ignited, the plasma jet (plume) is launched into the ambient through the nozzle of the tube. The kind of discharge 
(DBD) used there generates low-temperature plasmas that are suitable for the atomization of volatile species and can also be served as a ionization source for ambient mass spectrometry and ion mobility spectrometry [17-20]. The paper presents a source based on a plasma jet established at the end of a capillary DBD at atmospheric pressure and its application to mass spectrometry. Early results of spectroscopic analysis are given.

\section{Experimental arrangement}

The experimental configuration containing APPJ, high voltage power supply and control unit (oscilloscope) is schematically presented in Fig. 1. Power supply consists of two units: function generator and $\mathrm{HV}$ generator. As a high voltage generator piezoelectric transformer (PT) has been utilized giving up to $15 \mathrm{kV}\left(\mathrm{V}_{\mathrm{pp}}\right)$ with frequency of $70 \mathrm{kHz}$. Figure 2 shows the generator in question together with piezoelectric transformer outline.

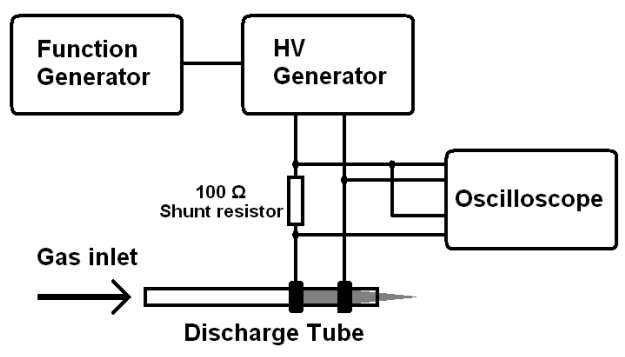

Fig. 1. Scheme of experimental configuration of atmospheric pressure plasma source based on DBD with HV power supply and control unit.

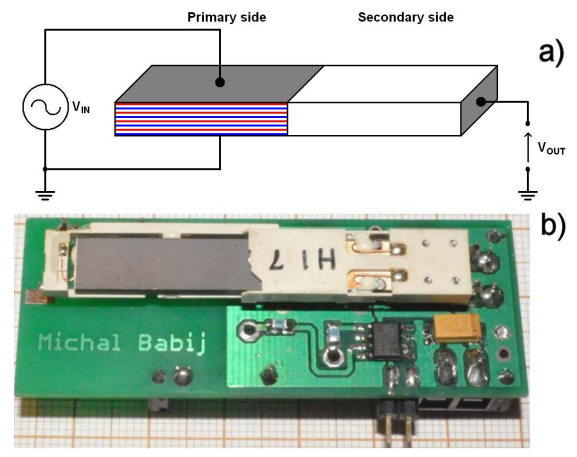

Fig. 2. High voltage generator with piezoelectric transformer (PT): (a) transformer outline, (b) PT as a part of $\mathrm{HV}$ generator.

The plasma jet was based on a capillary DBD and was realized using two kinds of glass capillaries. In the first stage of experiments soda glass tubes with outer diameter of $1.5 \mathrm{~mm}$ and inner diameter of $0.8 \mathrm{~mm}$ were examined. The electrodes of $2 \mathrm{~mm}$ width were made of copper rings surrounding the capillary tube, and the gap between the inner edges was $5 \mathrm{~mm}$. The grounded electrode was situated $5 \div 7 \mathrm{~mm}$ apart from the tube orifice. With the given electrode geometry plasma jet has been successfully launched with the use of noble gases (He and Ar). Unfortunately, it was stated that under high voltage and temperature an unexpected and disadvantageous phenomenon took place: glass electrolysis. That process together with various glass defects resulted in local overheating and destruction of the capillary in the vicinity of electrode edges.

To eliminate the plasma source disadvantages mentioned above, quartz capillaries with the same double electrode configuration for obtaining the DBD were applied and have been carefully investigated. With the given electrode material and geometry, i.e.:

a) thin layer electrodes (three layers: $\mathrm{Ni} / \mathrm{Cr}, \mathrm{Ni}$ and $\mathrm{Ag}$ ) deposited on the tube,

b) the same electrode width and separation as in the first design,

plasma jet can be successfully launched. The applied voltages $\left(\mathrm{V}_{\mathrm{pp}}\right)$ we adopted are below $12 \mathrm{kV}$ and the minimum value is down to $8 \mathrm{kV}$ for $\mathrm{He}$ and $11 \mathrm{kV}$ for Ar.

\section{Results}

The final version of atmospheric pressure plasma source based on DBD together with the generated plasma plume contacting a human finger are depicted in Fig. 3. The plasma jet formed by the device is stable and can be touched with a bare hand or passed over human skin without thermal damage. In general the specific heats of cells are larger than that of e.g. aluminum and tissues have larger heat capacitances than the aluminum thin film [21].

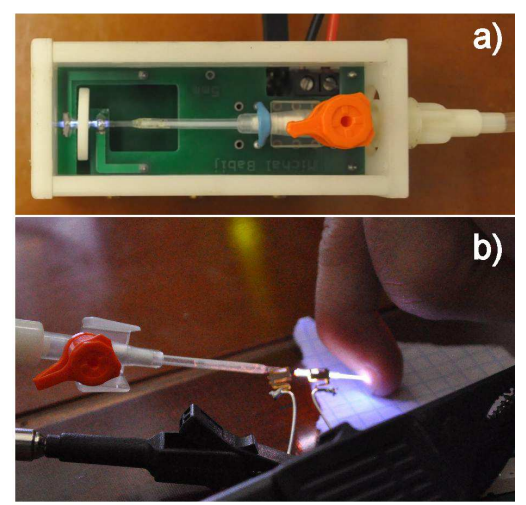

Fig. 3. Final version of plasma source based on DBD: (a) the upper view, (b) the plasma plume generated by the source contacting a human finger.

To evaluate the usefulness of our plasma jet in mass spectrometry, set of experiments have been done. The fact of having high mass resolution and accurate mass information has been examined for DBD ionization of caffeine. Its dilution was sprayed in the space between the 


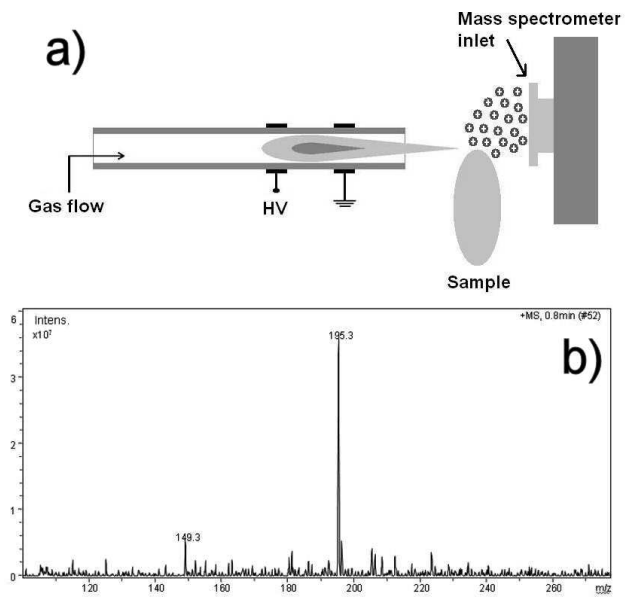

Fig. 4. Mass spectrometry with the APPJ as ionization source: (a) scheme of the experimental arrangement, (b) mass spectrum of caffeine (the signal at $\mathrm{m} / \mathrm{z}$ 195.3).

source and mass spectrometer. The signals $m / z$ were assigned as followed by the exact mass obtained by the mass spectrometer (Bruker Esquire 3000). A section of mass spectrum together with the scheme of the experimental arrangement is displayed in Fig. 4. Other substance examined was analgesic medicine "APAP Noc" (results will be published soon).

\section{Conclusion}

The paper presents a source (made in Wrocław University of Technology) based on a plasma jet established at the end of a capillary DBD at atmospheric pressure. The structure of the jet generator consists of piezoelectric transformer and two concentric and symmetric electrodes, between which the working gas flows at definite rate. The plasma plume formed outside the electrode region provides spatial separation of the plasma generation and surface processing regions. Together with source description early results of its potential application to mass spectrometry are presented.

The results of experiments obtained with the use of our jet were verified by reference plasma source (in AGH University of Science and Technology, Kraków). It was stated that sensitivity of both plasma sources was comparable and that the same substances were detected by mass spectrometer. Our APPJ has been demonstrated to be useful ionization instrument for mass spectrometry. Since some problems remain to be solved, next experiments are still under way in our group. The focus of work in the near future will be on experimental setup that has to be optimized and on detailed mass spectroscopic investigations.

\section{References}

[1] S.A. Starostin, P. Antony Premkumar, M. Creatore, H. de Vries, R.M.J. Paffen, M.C.M. van de Sanden, Appl. Phys. Lett. 96, 061502 (2010).

[2] M.C. Kim, D.K. Song, H.S. Shn, S.-H. Baeg, G.S. Kim, J.-H. Boo, J.G. Han, S.H. Yang, Surf. Coat. Technol. 171, 312 (2003).

[3] S.A. Fernández-Gutierrez, P.D. Pedr, M.J. Pitts, J. Powers, IEEE Trans. Plasma Sci. 38, 957 (2010).

[4] G.B. McCombs, M.L. Darby, Int. J. Dent. Hygiene 8, 10 (2010).

[5] M. Vandamme, E. Robert, S. Lerondel, V. Sarron, D. Ries, S. Dozias, J. Sobilo, D. Gosset, C. Kieda, B. Legrain, J.-M. Pouvesle, A. Le Pape, Int. J. Cancer 130, 2185 (2012).

[6] A. Schütze, J.Y. Jeong, S.E. Babayan, J. Park, G.S. Selwyn, R.F. Hicks, IEEE Trans. Plasma Sci. 26, 1685 (1998).

[7] A. Bogaerts, E. Neyts, R. Gijbels, J. van der Mullen, Spectrochim. Acta B 57, 609 (2002).

[8] V. Nehra, A. Kumar, H.K. Dwivedi, Int. J. Eng. 2, 53 (2007).

[9] N. Jiang, A.L. Ji, Z.X. Cao, J. Appl. Phys. 106, 013308 (2009).

[10] W.-C. Zhu, Q. Li, X.-M. Zhu, Y.-K. Pu, J. Phys. D, Appl. Phys. 42, 202002 (2009).

[11] J. Franzke, Anal. Bioanal. Chem. 395, 549 (2009).

[12] J. Ran, H. Luo, X. Wang, in: 30th Int. Conf. Phen. Ion. Gas, ICPIG 2011, Queen's University Belfast, Belfast 2011.

[13] X.P. Lu, Z.H. Jiang, Q. Xiong, Z.Y. Tang, Y. Pan, Appl. Phys. Lett. 92, 151504 (2008).

[14] A. Shashurin, M. Keidar, S. Bronnikov, R.A. Jurjus, M.A. Stepp, Appl. Phys. Lett. 93, 181501 (2008).

[15] Y.C. Hong, W.S. Kang, Y.B. Hong, W.J. Yi, H.S. Uhm, Phys. Plasmas 16, 123502 (2009).

[16] Y.S. Seo, H.W. Lee, H.C. Kwon, J. Choi, S.M. Lee, K.C. Woo, K.T. Kim, J.K. Lee, Thin Solid Films 519, 7071 (2011).

[17] J. Franzke, K. Kunze, M. Miclea, K. Niemax, J. Anal. At. Spectrom. 18, 802 (2003).

[18] A. Michels, S. Tombrink, W. Vautz, M. Miclea, J. Franzke, Spectrochim. Acta B 62, 1208 (2007).

[19] S.B. Olenici-Craciunescu, A. Michels, C. Meyer, R. Heming, S. Tombrink, W. Vautz, J. Franzke, Spectrochim. Acta B 64, 1253 (2009).

[20] S.B. Olenici-Craciunescu, S. Müller, A. Michels, V. Horvatic, C. Vadla, J. Franzke, Spectrochim. Acta B 66, 268 (2011).

[21] K.G. Kim, G.Y. Kim, Y.C. Hong, S.S. Yang, Microelectron. Eng. 87, 1177 (2010). 